\title{
Marine diesel engine - a novel approach of indicated power determination and related uncertainty level
}

A proposal of a new method for marine engine indicated power determination, based on direct piston position, instead of crankshaft angular position measurement, was presented. With the aid of bond graph theory, it was proved, that the new method is equivalent to conventional method. To verify hypothesis, that the new method has potential of determined indicated power uncertainty reduction, simulated calculations were carried out. Results of calculations for two marine diesel engines: medium-speed, four stroke and slowspeed two stroke type, at assumption of engine crankshaft constant speed, were compared with results obtained by a conventional method. Data, collected by means of a typical portable, industrial, digital indicator on engines in service, were used for simulation. The uncertainties of engines indicated power determined by means of two methods were analysed. The indicated power uncertainty, achieved for conventional method, was nearly twice higher than for new proposed method. Finally, uncertainty structure was analysed and evaluated for both: new and conventional method of engine indicated power determination.

Key words: marine diesel engine, indicated power, uncertainty

\section{Introduction}

High demand to improve fuel economy and exhaust gas emission characteristics requires a new technology for engine control. Also, increased expectation of marine diesel engines reliability calls for sophisticated control and development of engine diagnostics systems. Computational modelling of crank shaft position and rotational speed fluctuations could be used as a basis for engine diagnosis. Certain parameters were found, such as crank friction or instantaneous engine speed that provide a better basis for fault engine assessment [4]. Also, balanced cylinder combustion is important for reliable, emission-compliant engine operation. Throughout the years, several engine balance techniques have been developed and efficiently utilized. Unfortunately, no clear-cut standard has been established for them. Combustion pressure is analysed for specific quantitative values like indicated mean effective pressure, maximum pressure, firing pressure angle, cycle variations, etc. and certain events location (valve closure and opening, peak rate of pressure rise, etc.), as well as process variation between cylinders. However, the principle aim is indicated power estimation for each cylinder and consequently total engine indicated power assessment. Engine cylinder pressure analysis may be used to balance and tune the engine: e.g. valve and fuel injection timing as well as fuel bulk and compression pressure.

Usually, low speed, large bore diesel engine is applied as a vessel's main propulsion and stays in a range of power from a few thousand kilowatts to over $80 \mathrm{MW}$. The main engine is one of the most expensive functional components of the ocean-going vessel. Daily fuel consumption of the most powerful marine engines may exceed 250 tons. Therefore, such responsible machinery should be monitored and controlled adequately. Any main engine malfunction involves enormous costs of excessive fuel consumption, repairs and even bigger costs of ship's trip delays and lost freight. One of the solutions used to meet these requirements is a real-time cylinder pressure monitoring system. Gas pressure appraisal in combustion chamber of recipro- cating engine is mostly affected by two phenomena: the first of them are acoustic vibrations in a long duct, connecting combustion chamber with the sensor, the second is unreliable synchronization with the piston position. While the negative influence of acoustic vibrations can be solved by signal filtration with acceptable result, the problem of synchronization has not been solved yet satisfactorily. Instantaneous engine crankshaft rotational speed varies during the whole engine cycle. There are several possible ways of piston position estimation, presented in literature [1-3, $12,13]$. The majority of methods do not require a specific sensor placed inside the engine crankcase, but are based on the engine speed - measured by the typical pick-up mounted at flywheel. Supportive analysis of the cylinder gas pressure course $[8,12]$ is utilized too.

In order to monitor the overall engine output, the torque meter can be applied on the ship's main propulsion shaft. However, the determination of the indicated power in each cylinder unit brings more benefits indeed. In addition to the engine total indicated power, the balance of the engine load distribution between cylinder units needs to be evaluated too.

The indicated power is defined as a ratio of the indicated work generated in the single cycle to the time needed to generate that work [5]:

$$
P_{i}=\frac{L_{i}}{\tau_{c}}=\frac{L_{i} \cdot \omega}{2 \cdot n_{R} \cdot \pi}
$$

Generally, the principle of the indicated power determination strictly relies on the definitional formula (1). The method of indicated power determination has not changed much since the time of steam engines. Basically, the pressure course is integrated over the cylinder volume in order to determine the generated work per cycle and then the indicated power is determined. That solution comes directly from the classic definition and availability of engine cylinder gas pressure and crankshaft speed. Unfortunately, this method is still not sufficiently accurate in terms of modern requirements. One of the main reason is well-known prob- 
lem of the engine's piston top dead center (TDC) determination, during combustion pressure measurement [6-10]. Position of the TDC is essential for proper assigning of the positive or negative value to the incremental indicated work that is calculated. In various publications it is estimated that $1^{\circ}$ of a crank angle (CA) error in piston TDC determination, may lead to $3-10 \%$ of the determined indicated power inaccuracy [6-8]. As presented in [7], the TDC position standard uncertainty of $0.6^{\circ} \mathrm{CA}$ is achivable in a typical industrial conditions. There is a number of various methods aimed to increase the accuracy of pressure course with piston position synchronisation. Generally, they may be divided into two groups: pre measurement methods and post measurement methods. Installation of incremental encoders on the crankshaft may be an example of the first group. Also application of finite element method (FEM), for crankshaft torsional or vibratory deformation evaluation, is a kind of pre measurement method for increasing the accuracy of indicated power determination. The second group representative example is a rate of pressure rise $\mathrm{dp} / \mathrm{d} \alpha$ analysis. Unfortunatelly, presented exapmles provide limited improvement only, or they are selected for specific engines constructions or working conditions. Incremental encoders allow to reduce greatly the irregular shaft speed influence, however the problem of their static adjustment still remains unsolved. The method based on FEM analysis, requires detailed informations about the shaftline geometry, material and set of initial and boundary conditions data. Besides, enormous computational capacity is to be engaged for such model calculations, that makes it unavailable on board the vessel.

The concept of the thermodynamic TDC, understood as the one, detected by pressure course or heat flow analysis, was proposed [8, 12, 13]. However, the thermodynamic TDC differs from the real TDC $[6,8,12,13]$, by an angle $\varphi$, which is difficult to evaluate usually. As the main reasons, the heat exchange with cooled, engine chamber and loss of charge through the piston rings locks, are indicated $[6,13]$. The $\mathrm{dp} / \mathrm{d} \alpha$ analysis gives quite satisfactory results for slow speed engines, where ignition occures after the piston TDC, however for medium speed engines, where ignition occures before piston TDC it is much less accurate.

Currently used method of indicated power determination exhibits one more disadvantage - difficulties with precise uncertainty estimation. Actually none of the commercially available, industrial indicators is equipped with manufacturer's specification regarding accuracy of calculated indicated power. Calibration certificate of the pressure sensor is attached eventually. This is mostly due to the complexity of the indicated power uncertainty evaluation and lack of standardised procedures [6, 7]. A proposal of a method for the indicated power uncertainty evaluation was presented in [7].

This paper is meant as a proposal of a method for indicated power determination, free of a major factor influencing the overall indicated power uncertainty - the TDC determination uncertainty.

\section{Proposed method fundamentals}

The problem of the piston engine's indicated power determination can be examined in terms of the bond graphs theory as a rate of energy transfer [9]. The bond graph representation of the piston engine's kinematics is shown on Figure 1.

$$
\mathrm{CC} \frac{\mathrm{p}}{\dot{\mathrm{V}}} \mathrm{GY} \frac{\mathrm{F}_{\mathrm{g}}}{\mathrm{v}} \mathrm{CM}
$$

Fig. 1. Cylinder, piston and crankshaft assembly represented by a bond graph [9]

The power of the working gas in combustion chamber (CC) volume is described by a product of cylinder gas pressure $\mathrm{p}$ and rate of $\mathrm{CC}$ volume $\dot{\mathrm{V}}$. The piston appears to be a bond graph node called gyrator (GY), where the rate of energy flow undergoes transformation from the energy of the working gas pressure into mechanical energy of the moving crank assembly. Following gyrator, the rate of energy transfer is described by a product of the force $F_{g}$, exerted on piston by working gas pressure, and piston speed $\mathrm{v}$.

Consequently, the rate of energy transfer to the engine's crank mechanism CM and later, through engine's coupling, to the energy consumer. Assuming, that the rate of energy flow is transformed in the gyrator in a lossless process, it can be described by equation (2):

$$
\mathrm{p} \cdot \dot{\mathrm{V}}=\mathrm{F}_{\mathrm{g}} \cdot \mathrm{V}
$$

For given combustion chamber geometry, known cylinder gas pressure and piston speed, the formula (3) for instant rate of energy transfer may be applied:

$$
\frac{d E(t)}{d t}=F_{g}(t) \cdot v(t)=A \cdot p(t) \cdot \frac{d S(t)}{d t}
$$

By integration, with respect to time, the indicated work of a cycle may be determined:

$$
\int_{0}^{\tau_{c}} \frac{d E(t)}{d t} d t=A \int_{0}^{\tau_{c}} p(t) \cdot \frac{d S(t)}{d t} d t=L_{i}
$$

Equations (3) and (4) prove that the indicated work may be evaluated from known cylinder gas pressure $\mathrm{p}$, the piston speed $\mathrm{v}$ and piston area $\mathrm{A}$. Consequently, the indicated power for one cylinder may be determined (5):

$$
P_{i}=\frac{1}{\tau_{c}} \int_{0}^{\tau_{c}} \frac{d E(t)}{d t} d t=\frac{A}{\tau_{c}} \cdot \int_{0}^{\tau_{c}} p(t) \cdot \frac{d S(t)}{d t} d t
$$

Specific technical means to observe the piston movement should be secured in order to apply the proposed method of the indicated power determination. Direct measurement of the piston speed might be problematic. A better solution is to utilise a number of known piston positions. From a series of recorded pairs: piston position and time, a function $\mathrm{S}(\mathrm{t})$, describing piston shift over the time can be elaborated and, consequently, piston velocity function $\mathrm{v}(\mathrm{t})$ can be established. Effectively, presented technique may be called as an observation method of the indicated power determination.

\section{The uncertainty analysis}

The overall uncertainty of the determined indicated power depends on the individual uncertainty components in equation (5). The piston area uncertainty as well as cylinder gas pressure measurement uncertainty are the same in new observation method as in the well-known conventional 
method, based on the classic definition. The piston area $\mathrm{A}$ standard uncertainty type B is found from equation (6):

$$
u_{B}(A)=\frac{\partial A}{\partial D_{n}} \cdot u_{B}\left(D_{n}\right)=\frac{\pi \cdot D_{n}}{2} \cdot u_{B}\left(D_{n}\right)
$$

The dynamic pressure measurement accuracy problem is common for both methods, it is well described in literature $[5,6,13]$. It should be analysed in the same way regardless to the indicated power determination method applied. Consequently, pressure uncertainty has no influence on the difference in indicated power uncertainty between both described methods. To simplify analysis of the proposed method, which is concentrated on TDC determination issue, pressure measurement uncertainty is reduced to a single sample uncertainty which can be found from formula (7):

$$
\mathrm{u}_{\mathrm{B}}(\mathrm{p})=\frac{\mathrm{R} \cdot \Delta \mathrm{p}}{100 \% \cdot \sqrt{3}}
$$

Typically, measured data are recorded at a fixed sampling rate. The relative uncertainty of the time interval measurement $u_{r}(d t)$ is quantitatively identical with the sampling rate uncertainty and usually given in the analogue-digital converter specification. Typical acquisition cards are equipped with clocks of basic frequency $f_{b}=5-10$ $\mathrm{MHz}$ and relative stability of $100 \mathrm{ppm}$, that means: $\mathrm{u}_{\mathrm{r}}\left(\mathrm{f}_{\mathrm{b}}\right)=$ $\pm 0.01 \%$.

In an industrial measuring devices, the typical sampling rate is $10-25 \mathrm{kHz}$, to secure sufficient time resolution of the measurement. The clock time interval uncertainty can be determined by means of the sampling rate parameters:

$$
\mathrm{u}_{\mathrm{B}}(\mathrm{dt})=\frac{1}{\mathrm{f}_{\mathrm{s}}}-\frac{1}{\mathrm{f}_{\mathrm{s}}+\mathrm{f}_{\mathrm{s}} \cdot \mathrm{u}_{\mathrm{r}}\left(\mathrm{f}_{\mathrm{b}}\right)}
$$

Assuming the sampling frequency of $\mathrm{f}_{\mathrm{s}}=10 \mathrm{kHz}$, thus for typical industrial application the clock time interval uncertainty amounts to $\mathrm{u}_{\mathrm{B}}(\mathrm{dt})=10 \mathrm{~ns}$.

The problem of the piston position uncertainty determination is closely related to the selected measurement technique. The review of linear position sensors available on the market allows for assumption that the measurement uncertainty of $\mathrm{u}_{\mathrm{B}}(\mathrm{S})=0.1 \mathrm{~mm}$ is available. The finite number of piston positions $S_{i}$ measured in the time domain can be approximated by a continuous function $\widehat{S}(\mathrm{t})$. The overall uncertainty $u_{B}\left(S_{p}\right)$ of the piston position estimation (9) may be derived from the error propagation law as a singular value measurement uncertainty $\mathrm{u}_{\mathrm{B}}(\mathrm{S})$ and the approximating function $\widehat{S}(\mathrm{t})$ mean square error which is a direct estimator of a fitting quality:

$$
\mathrm{u}_{\mathrm{B}}\left(\mathrm{S}_{\mathrm{p}}\right)=\sqrt{\mathrm{u}_{\mathrm{B}}^{2}(\mathrm{~S})+\mathrm{s}^{2}(\widehat{\mathrm{S}}(\mathrm{t}))}
$$

In time interval dt between consecutive samples $\mathrm{i}$ and $\mathrm{i}+1$ piston displaces the distance:

$$
\mathrm{dS}_{\mathrm{i}}=\mathrm{S}_{\mathrm{i}+1}-\mathrm{S}_{\mathrm{i}}
$$

The uncertainty of the distance $\mathrm{dS}_{\mathrm{i}}(10)$ is a function of both boundary positions $S_{i}$ and $S_{i+1}$ uncertainties. For a set of consecutive distances $\mathrm{dS}_{\mathrm{i}}$, a set $\mathrm{A}_{\mathrm{S}}$ of starting positions $S_{i} \in A_{S}$ can be defined. Similarly, a set $B_{S}$ of ending positions $S_{i+1} \in B_{S}$ can be defined too. Correlation coefficient
$r\left(A_{S}, B_{S}\right)$ of both sets: $A_{S}$ and $B_{S}$ can be calculated accordingly. Subsequently the uncertainty of a distance $\mathrm{dS}_{\mathrm{i}}$ can be determined form the formula for correlated values [10] (11):

$$
\begin{gathered}
\mathrm{U}_{\mathrm{B}}(\mathrm{dS})= \\
\sqrt{\mathrm{u}_{\mathrm{B}}^{2}\left(\mathrm{~S}_{\mathrm{p}}\right)+\mathrm{u}_{\mathrm{B}}^{2}\left(\mathrm{~S}_{\mathrm{p}}\right)-2 \cdot \mathrm{u}_{\mathrm{B}}\left(\mathrm{S}_{\mathrm{p}}\right) \cdot \mathrm{u}_{\mathrm{B}}\left(\mathrm{S}_{\mathrm{p}}\right) \cdot \mathrm{r}\left(\mathrm{A}_{\mathrm{S}}, \mathrm{B}_{\mathrm{S}}\right)}
\end{gathered}
$$

Since the piston position is described by means of a continuous approximating function $\widehat{S}(\mathrm{t})$, the piston's speed function $\mathrm{v}(\mathrm{t})$ can be determined:

$$
v(t)=\frac{d \hat{s}(t)}{d t}
$$

The piston speed uncertainty can be determined with the aid of the formula (13):

$$
\mathrm{u}_{\mathrm{B}}(\mathrm{v}(\mathrm{t}))=\sqrt{\left(\frac{\partial \mathrm{v}(\mathrm{t})}{\partial \mathrm{dt}} \cdot \mathrm{u}_{\mathrm{B}}(\mathrm{dt})\right)^{2}+\left(\frac{\partial \mathrm{v}(\mathrm{t})}{\partial \mathrm{d} \hat{\mathrm{S}}(\mathrm{t})} \cdot \mathrm{u}_{\mathrm{B}}(\mathrm{dS})\right)^{2}}
$$

Once the piston speed and cylinder gas pressure uncertainties are known, the rate of the energy flow uncertainty can be evaluated. Based on equations (3), (6), (7) and (13), the equation can be formulated:

$$
\mathrm{u}_{\mathrm{B}}(\dot{\mathrm{E}})=\sqrt{\left(\frac{\partial \dot{\mathrm{E}}}{\partial \mathrm{A}} \cdot \mathrm{u}(\mathrm{A})\right)^{2}+\left(\frac{\partial \dot{\mathrm{E}}}{\partial \mathrm{p}} \cdot \mathrm{u}(\mathrm{p})\right)^{2}+\left(\frac{\partial \dot{\mathrm{E}}}{\partial \mathrm{v}} \cdot \mathrm{u}(\mathrm{v})\right)^{2}}
$$

In the presented method, to evaluate the cycle work, the rate of energy flow $\dot{E}$ must be integrated over the time (4). This is done by means of the numerical trapezoidal method usually. For the uncertainty determination, an uncertainty of the mean value of two consecutive values $\dot{\mathrm{E}}_{\mathrm{i}}$ and $\dot{\mathrm{E}}_{\mathrm{i}+1}$ should be determined first. The formula (15) for correlated input data can be applied, accordingly [10]. A set $A_{E}$ of preceeding values $\dot{E}_{i} \in A_{E}$ and a set $B_{E}$ of following values $\dot{\mathrm{E}}_{\mathrm{i}+1} \in \mathrm{A}_{\mathrm{E}}$ were defined and then a correlation coefficient $r\left(A_{E}, B_{E}\right)$ was calculated.

$$
\begin{gathered}
\mathrm{U}_{\mathrm{B}}\left(\dot{\mathrm{E}}_{\mathrm{av}}\right)= \\
\sqrt{\mathrm{u}_{\mathrm{B}}^{2}\left(\dot{\mathrm{E}}_{\mathrm{i}}\right)+\mathrm{u}_{\mathrm{B}}^{2}\left(\dot{\mathrm{E}}_{\mathrm{i}+1}\right)+2 \cdot \mathrm{u}_{\mathrm{B}}\left(\dot{\mathrm{E}}_{\mathrm{i}}\right) \cdot \mathrm{u}_{\mathrm{B}}\left(\dot{\mathrm{E}}_{\mathrm{i}+1}\right) \cdot \mathrm{r}\left(\mathrm{A}_{\mathrm{E}}, \mathrm{B}_{\mathrm{E}}\right)}
\end{gathered}
$$

For numerical integration, the elementary work is to be determined initially, its uncertainty can be expressed as:

$$
\mathrm{u}_{\mathrm{B}}(\dot{\mathrm{E}} \mathrm{dt})=\sqrt{\left(\dot{\mathrm{E}}_{\mathrm{av}} \cdot \mathrm{u}_{\mathrm{B}}(\mathrm{dt})\right)^{2}+\left(\mathrm{dt} \cdot \mathrm{u}_{\mathrm{B}}\left(\dot{\mathrm{E}}_{\mathrm{av}}\right)\right)^{2}}
$$

In consequence, the uncertainty of the indicated work of a single cycle can be defined from the equation:

$$
\mathrm{u}_{\mathrm{B}}\left(\mathrm{L}_{\mathrm{i}}\right)=\sqrt{\sum_{\mathrm{j}=1}^{\mathrm{j}=\mathrm{k}} \mathrm{u}_{\mathrm{B}}^{2}\left(\dot{\mathrm{E}}_{\mathrm{j}} \mathrm{dt}\right)}
$$

Finally, the single cycle indicated power uncertainty is assessed as follows: 


$$
\mathrm{u}_{\mathrm{B}}\left(\mathrm{P}_{\mathrm{i}}\right)=\sqrt{\left(\frac{\partial \mathrm{P}_{\mathrm{i}}}{\partial \tau_{\mathrm{c}}} \cdot \mathrm{u}_{\mathrm{B}}(\mathrm{dt})\right)^{2}+\left(\frac{\partial \mathrm{P}_{\mathrm{i}}}{\partial \mathrm{L}_{\mathrm{i}}} \cdot \mathrm{u}_{\mathrm{B}}\left(\mathrm{L}_{\mathrm{i}}\right)\right)^{2}}
$$

Final indicated power uncertainty equation (18) contains less variables and is much More convenient for a new approach, comparing to the conventional method [7] of indicated power determination. On the contrary to the conventional method [7], there are no factors, like a TDC sensor positioning, with unknown or difficult to evaluate uncertainties too. Consequently, a hypothesis may be formulated, that the new method might lead to the indicated power determination with lower uncertainty comparing to conventional method.

\section{The simulation results discussion}

The above presented uncertainty analysis of the proposed method was a subject to the validation test against the conventional method. The test was designed as a simulation calculation with the utilisation of data recorded by means of conventional method. The main goal of the validation was the comparison of indicated power and its uncertainty, resulted from common input but estimated with different methods. The original data sets for simulation calculations were collected for two marine diesel engines:

- medium-speed, four stroke - Hyundai Himsen H25/33,

- slow-speed, two stroke - MAN K90MC-C.

Both engines were in good service condition. The fuel injection valves were in the middle of their recommended working period. The exhaust gas temperatures, cylinder compression and maximum pressures did not indicate any related malfunctions to the leaky exhaust valves or piston rings.

Cylinder process data sets were collected by means of typical industrial digital indicator Premet $\mathrm{XL}^{\circledR}$ of Lemag $\mathrm{GmbH}$, during engines service operation. Engines were operated for at least 1 hour at constant load before measurements were carried out. During measurements, engines load remained constant and several monitored operational parameters, like charging air pressure, proved it. The slow speed engine was equipped with the LEMAG Multiscan Sensor $^{\circledR}$, which is a type of incremental encoder, installed on the propulsion shaft. The sensor resolution is 360 pulses per revolution, that allows for substantial reduction of the shaft speed irregularity influences. The medium speed engine TDC was indicated by an inductive pick-up installed over a flywheel. Both: multiscan sensor and inductive sensor, prior to the measurement, were adjusted statically to the TDC of piston in cylinder unit No. 1, the closest one to the flywheel. The indicator presents the recorded set of measured data as an average pressure course calculated from the initially pre-set number of engine cycles. Even though it is a good practice to use the highest available number of cycles for average calculation, specifically for engines directly driving ship's propeller, too long time of indication increases the risk of recording engine load fluctuation due to influence of the sea swell. In case of engine driving electric generator, in industrial condition, there is similar risk caused by higher possibility of unexpected start or stop of a large electric consumer. For those reasons, the amount of observed cycles pre-set was chosen as 10 for every monitored engine. Utilised digital indicator allows only for post measurement TDC correction by means of pressure rise $\mathrm{dp} / \mathrm{d} \alpha$ analysis. However, such analysis was unreliable, specifically for medium speed engine due to advanced ignition. For this reason, it was decided to utilise uncorrected data for both engines. The influence of the pressure measurement uncertainty on the overall indicated power uncertainty was the same for both methods, therefore this complicated analysis was reduced to the sensor range and linearity only. Disturbances in the pressure measurements are independent on the indicated power determination method and therefore may be examined separately.

Recorded data sets were used to compare results of observation technique calculation, marked as method A, with outcomes given by the industrial indicator, which result was marked as method B. For further analysis and calculations only the single cylinder unit No. 1 data of each engine were considered. In both cases, it was assumed that the crankshaft angular speed was constant. This assumption was justified in case of slow speed engine due to installed incremental encoder, allowing to neglect the shaft rotational speed irregularity. The medium speed engine crankshaft speed irregularity caused a minor influence on the resulted indicated power uncertainty [7]. Additionally, this assumption allowed for an easier comparison of the uncertainty achieved. It is expected that the proposed method A, based on direct piston observation, should not be prone to the speed irregularity influence, while for measured engines this influence is limited. Finally, the indicated power and related uncertainties evaluations for both methods were carried out and presented in Table 1. Exclusively, in case of method B the uncertainties were evaluated by means of method proposed in [7]. The TDC positioning uncertainties were evaluated for typical industrial conditions.

Table 1. Results of indicated work and power calculations with their uncertainties

\begin{tabular}{|l|c|c|c|c|}
\hline Engine type & \multicolumn{2}{|c|}{ Hyundai H25/33 } & \multicolumn{2}{c|}{ MAN K90 MC-C } \\
\hline Load & \multicolumn{2}{|c|}{$59 \%$} & \multicolumn{2}{c|}{$80 \%$} \\
\hline Method & A & B & A & B \\
\hline $\begin{array}{l}\text { TDC uncertain- } \\
\text { ty [7] }\end{array}$ & - & $0.63^{\circ} \mathrm{CA}$ & - & $0.57^{\circ} \mathrm{CA}$ \\
\hline $\mathrm{P}_{\mathrm{i}}$ & $189 \mathrm{~kW}$ & $182 \mathrm{~kW}$ & $3991 \mathrm{~kW}$ & $3850 \mathrm{~kW}$ \\
\hline $\mathrm{u}_{\mathrm{B}}\left(\mathrm{P}_{\mathrm{i}}\right)$ & $\begin{array}{r} \pm 4.4 \mathrm{~kW} \\
( \pm 2.3 \%)\end{array}$ & $\begin{array}{l} \pm 12 \mathrm{~kW} \\
( \pm 6.8 \%)\end{array}$ & $\begin{array}{c} \pm 86 \mathrm{~kW} \\
( \pm 2.1 \%)\end{array}$ & $\begin{array}{c} \pm 180 \mathrm{~kW} \\
( \pm 4.8 \%)\end{array}$ \\
\hline
\end{tabular}

For uncertainty determination, several values, listed in Table 2, had to be assumed. In case of time interval dt and pressure sensor range $\mathrm{R}$, typical values of the industrial systems were accepted. Piston position approximating function $\widehat{S}(t)$ was assumed with poor fitting quality, even if hypothetical sensor class, represented by the singular value measurement uncertainty $\mathrm{u}_{\mathrm{B}}(\mathrm{S})$, would exhibit higher precision.

Table 2. Basic assumptions for the observation method uncertainty evaluation

\begin{tabular}{|l|c|}
\hline $\mathrm{uB}(\mathrm{dt})$ & $10 \mathrm{~ns}$ \\
\hline $\mathrm{uB}(\mathrm{S})$ & $0.1 \mathrm{~mm}$ \\
\hline $\mathrm{s}(\widehat{\mathrm{S}}(\mathrm{t}))$ & $4 \mathrm{~mm}$ \\
\hline $\mathrm{R}$ & $25 \mathrm{MPa}$ \\
\hline
\end{tabular}


The uncertainty distribution for the indicated power determination using methods $\mathrm{A}$ and $\mathrm{B}$, of both marine engines are presented in Figs 1 and 2. The uncertainty of indicated power determined by means of the proposed technique $-\mathrm{A}$, is considerable smaller than uncertainty established using the conventional method $-\mathrm{B}$. The improvement is significant, approximately up to three times for medium speed engine and up to two times for slow-speed K90MC-C engine. In both methods, $\mathrm{A}$ and $\mathrm{B}$ the pressure measurement uncertainty is settled as main component. However, in case of a conventional method - B also other issues, described in details in [7], have a significant influence on the overall indicated power uncertainty.
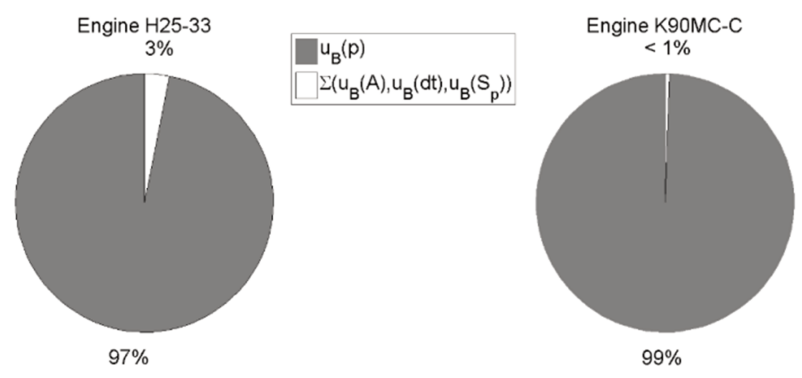

Fig. 1. Distribution of the indicated power uncertainties (method A) for marine engines - medium speed $\mathrm{H} 25-33$ and slow speed K90MC-C; $u_{B}(p)$ - cylinder gas pressure uncertainty, $\mathrm{u}_{\mathrm{B}}(\mathrm{A})$ - piston surface uncertainty, $\mathrm{u}_{\mathrm{B}}(\mathrm{dt})$ - clock time interval uncertainty; $\mathrm{u}_{\mathrm{B}}(\mathrm{S})$ - piston position uncertainty
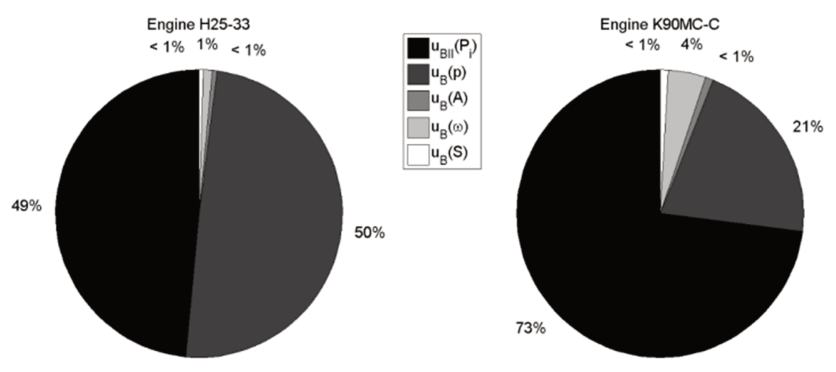

Fig. 2. Distribution of the indicated power uncertainties (method B) for marine engines - medium speed H25-33 and slow speed K90MC-C [7]; $\mathrm{u}_{\mathrm{BII}}\left(\mathrm{P}_{\mathrm{i}}\right)$ - indicated power uncertainty type $\mathrm{B}$ kind II, $\mathrm{u}_{\mathrm{B}}(\mathrm{p})$ - cylinder gas pressure uncertainty, $u_{B}(A)$ - piston surface uncertainty, $u_{B}(\omega)$ - crank-

shaft angular speed uncertainty; $\mathrm{u}_{\mathrm{B}}(\mathrm{S})$ - piston position uncertainty

Generally, the proposed method displays main component of the overall uncertainty as the pressure measurement uncertainty, with contribution of $97 \%$ to $99 \%$. That makes a significant quality difference comparing to the conventional method. Also, the number of important factors is seriously reduced, that simplifies the uncertainty evaluation. However, it should be underlined that presented technique comprises essential assumptions: piston position samples are uniformly distributed and recorded over the entire stroke, and crankshaft rotational speed is constant over the entire cycle. In fact, the crankshaft speed irregularities [11] combined with the piston position observation difficulties will not allow for such optimistic result.

In the presented simulation, in case of both methods A and $B$, the uncertainty of type A [10] could not be evaluated due to lack of repeatedly collected data. It is expected however that, in case of the observation method, the value of type A uncertainty is significant. Principally, indicated power uncertainty type A is expected to include TDC position uncertainty, which is estimated on the base of the piston speed approximating function's $\mathrm{v}(\mathrm{t})$ zero-crossing point.

\section{Conclusions}

Basically, new calculation technique should demonstrate advantages against current approach and observation method presents such potential of reducing uncertainty of marine engine indicated power. Additionally, proposed method substantially improves uncertainty calculation predictability. In presented simulation confirmation of significant uncertainty level reduction was achieved. Main factor influencing the overall indicated power uncertainty is cylinder gas pressure measurement uncertainty, which is responsible for over $90 \%$ of the overall uncertainty value. That is a qualitative improvement in comparison to the conventional method, where some, crucial for uncertainty evaluation factors are difficult to measure and evaluate.

The functional representation of the piston speed allows determination of the instant piston speed exactly at a time of every pressure sample, therefore difficult process of the pressure course synchronization with crankshaft angle is no more required. Finally, direct piston position measurement and consequent piston speed determination do not require additional piston's TDC determination. The piston TDC is automatically detected at the speed function zero-crossing points. The calculated functions of piston speed $v(t)$ and the rate of energy transfer $\dot{E}(t)$ seems to be very promising as a future diagnostic and engine performance evaluation tools.

Practical introduction of the proposed method may pose a real problem. Not only the difficult environment inside of the crankcase must be considered. Fulfilling of safety requirements (potential oil mist ignition) and classification societies acceptance may be difficult to overcome. From the other hand, proximity sensors, for a bearing monitoring systems, are currently successfully installed in large crosshead engines. This type of marine engines seems to be especially fit for the proposed method application. Access to the crosshead, which is rigidly connected to the piston is easy, and it allows for entire piston stroke observation.

\section{Nomenclature}

A area of the engine piston $\left[\mathrm{m}^{2}\right]$

$A_{E} \quad$ set of incremental rate of energy flow preceeding values $\dot{\mathrm{E}}_{\mathrm{i}}$

$B_{E} \quad$ set of incremental rate of energy flow following values $\dot{\mathrm{E}}_{\mathrm{i}+1}$
$\mathrm{A}_{\mathrm{S}} \quad$ set of piston incremental travelling distances starting points $S_{i}$

$\mathrm{B}_{\mathrm{S}} \quad$ set of piston incremental travelling distances ending points $S_{i+1}$

CC combustion chamber 
CM crank mechanism

dt time interval [s]

$\mathrm{D}_{\mathrm{n}} \quad$ nominal cylinder bore $[\mathrm{m}]$

E energy [J]

$\dot{\mathrm{E}} \quad$ rate of the energy flow $[\mathrm{J} / \mathrm{s}]$

$\dot{\mathrm{E}}_{\mathrm{av}}$ average of the consecutive rate of the energy flow values $[\mathrm{J} / \mathrm{s}]$

$\mathrm{f}_{\mathrm{b}} \quad$ clock frequency $[\mathrm{Hz}]$

$\mathrm{F}_{\mathrm{g}} \quad$ piston gas force $[\mathrm{N}]$

$\mathrm{f}_{\mathrm{s}} \quad$ sampling frequency $[\mathrm{Hz}]$

GY gyrator

$\mathrm{k}$ number of samples per engine working cycle

$\mathrm{L}_{\mathrm{i}} \quad$ indicated work [J]

$\mathrm{n} \quad$ crank rotational speed [rps]

$\mathrm{n}_{\mathrm{R}} \quad$ number of crank revolutions per cycle

$\mathrm{r} \quad$ correlation coefficient
$\mathrm{R}$

$\mathrm{S}$

$\mathrm{S}$

$\widehat{\mathrm{S}}(\mathrm{t})$ approximating function of a piston position [m]

$\mathrm{p} \quad$ cylinder gas pressure $[\mathrm{Pa}]$

$\mathrm{P}_{\mathrm{i}} \quad$ indicated power $[\mathrm{W}]$

$\mathrm{u}_{\mathrm{A}} \quad$ uncertainty type $\mathrm{A}$

$\mathrm{u}_{\mathrm{B}} \quad$ uncertainty type $\mathrm{B}$

$\mathrm{u}_{\mathrm{r}} \quad$ relative uncertainty

$\mathrm{V} \quad$ piston speed $[\mathrm{m} / \mathrm{s}]$

$\dot{\mathrm{V}}$. rate of combustion chamber volume change $\left[\mathrm{m}^{3} / \mathrm{s}\right]$

$\Delta \mathrm{p} \quad$ pressure sensor's non-linearity error [\% FSO]

$\tau_{\mathrm{c}} \quad$ cycle time $[\mathrm{s}]$

$\omega \quad$ crankshaft angular speed $[\mathrm{rad} / \mathrm{s}]$

\section{Bibliography}

[1] WANG, Y., LIM, T.C. An analysis of modal damping sources in reciprocating engine. Journal of Sound and Vibration. 2001, 242(1), 179-193.

[2] NILSSON, M. Modelling flywheel-speed variations based on cylinder pressure [Master's thesis]. 2004, Linköping: Linköping University.

[3] TRAVER, M.L., ATKINSON, R.J., ATKINSON, C.M. Neural network-based diesel engine emissions prediction using cylinder gas combustion pressure. SAE Technical Paper. 1999, 1999-01-1532.

[4] TWIDDLE, J.A., JONES, N.B. A high-level technique for diesel engine combustion system condition monitoring and fault diagnosis. Proceedings of the Institution of Mechanical Engineers Part I: Journal of Systems and Control Engineering. 2002, 216(2), 125-134.

[5] HEYWOOD, J.B. Internal combustion engines fundamentals. Singapore, 1988. McGraw-Hill.

[6] BROWN, W.L. Methods for evaluating requirements and errors in cylinder pressure measurement SAE Technical Paper. 1967,670008 .

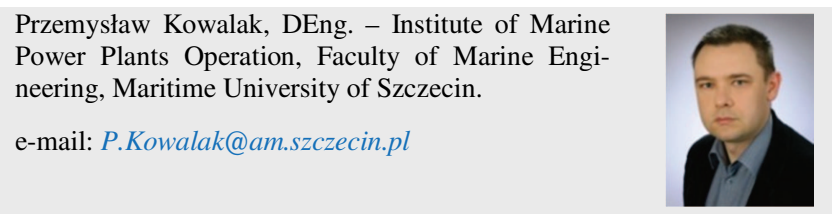

Tadeusz Borkowski, DSc., DEng. - Faculty of Marine Engineering, Maritime University of Szczecin.

e-mail: T.Borkowski@am.szczecin.pl
[7] KOWALAK, P. Marine engine indicated power uncertainty determination. Combustion Engines. 2015, 161(2), 3-13.

[8] STAŚ, M.J. An universally applicable thermodynamic method for T.D.C. determination. SAE Technical Paper. 2000, 2000-01-0561.

[9] CICHY, M. Silniki o działaniu cyklicznym podstawy teorii działania [Cyclic engines theory and fundamentals]. 1989, Gdańsk: Wydawnictwo Politechniki Gdańskiej.

[10] International Organization for Standardisation. 1995. Guide to the expression of uncertainty in measurement. Geneva: ISO.

[11] KOWALAK, P. Speed irregularity characteristic of low speed, two-stroke marine diesel engine applied as vessel's main propulsion. Journal of KONES Powertrain and Transport. 2008, 15(4), 263-268.

[12] TAZEROUT, M., LE CORRE, O., ROUSSEAU, S. TDC determination in IC engines based on the thermodynamic analysis of the temperature-entropy diagram. SAE Technical Paper. 1999, 1999-01-149.

[13] WIMMER, A., GLASER, J. Indykowanie silnika [Engine's indication]. 2004, Warsaw: Instytut Zastosowań Techniki.

Prof. Stefan Żmudzki, DSc., DEng. - Faculty of Marine Engineering, Maritime University of Szczecin.

e-mail: S.Zmudzki@am.szczecin.pl

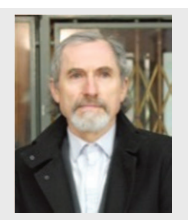

